\title{
Polarization of radiation of point-like source reflected from turbulent magnetized atmosphere
}

\author{
N. A. Silant'ev ${ }^{1}$ and Yu. N. Gnedin ${ }^{2}$ \\ ${ }^{1}$ Instituto Nacional de Astrofísica, Óptica y Electrónica, Luis Enrique Erro 1, Apartado Postal 51 y 216, 72840, Tonantzintla, Pue., \\ México \\ e-mail: silant@inaoep.mx \\ 2 Central Astronomical Observatory at Pulkovo of the Russian Academy of Sciences, 196140 St.-Petersburg, Pulkovo, Russia
}

Received 10 October 2007 / Accepted 7 December 2007

\begin{abstract}
We consider the multiple scattering of the light from a point-like source located above the semi-infinite electron, turbulent, and magnetized atmospheres. The frozen magnetic field has both the regular $\boldsymbol{B}_{0}$ and stochastic $\boldsymbol{B}^{\prime}$ components $\left(\boldsymbol{B}=\boldsymbol{B}_{0}+\boldsymbol{B}^{\prime}\right)$. The stochastic Faraday rotations due to fluctuations $\boldsymbol{B}^{\prime}$ decrease the intensity of each separate polarized beam (the extinction factor is proportional to $\left.\lambda^{4}\left\langle B^{\prime 2}\right\rangle\right)$. This decrease at large $\lambda$ dominates the usual decrease $\left(\propto \lambda^{2} B_{0} \cos \Theta_{0}\right)$ caused by summing beams with very different Faraday's rotation angles. This effect changes the spectrum of polarization degree as compared with what is influenced by the regular magnetic field. We calculated the integral (observed) polarization of the reflected radiation with the inclusion of unpolarized radiation going directly from the point-like source. We present the observed polarization for various degrees of true absorption of the radiation into the atmosphere and the values of magnetic energy fluctuations. The spectra of polarization in the optical $(\lambda=0-1 \mu \mathrm{m})$, infrared $(\lambda=1-5 \mu \mathrm{m})$, and X-ray $(E=1-50 \mathrm{keV})$ regions of the wavelengths are presented. We discuss the possibility of estimating parameters of magnetic field fluctuations from the observation of the spectra of polarization in AGNs with the X-ray excesses and in the turbulent accretion disk in NGC 4258.
\end{abstract}

Key words. radiative transfer - turbulence - magnetic fields - polarization

\section{Introduction}

Many astronomical objects - neutron stars, white dwarfs, active galactic nuclei, hot accretion disks, envelopes around of X-ray sources, and many usual stars (for example our Sun) have magnetic fields (see Blaes 2003). For a number of objects such as binary systems with optically thick accretion disks (see Cherepashchuk 2000) and flare stars (Grinin \& Domke 1971; Gurzadyan 1980), we observe the linear polarization in different regions of the wavelengths. There are the reasons to consider that this polarization stems in a number of cases from the reflection of unpolarized radiation of the point-like sources off in the optically thick accretion disks (see, for example, Czerny et al. 2004) or the atmospheres of the stars.

It is known (see, for example, Dolginov et al. 1995) that the Faraday rotation of the polarization plane in magnetized plasma disks and atmospheres can strongly diminish the degree of the outgoing radiation. Because the angle of the Faraday rotation is proportional to the square of wavelength (see below), the outgoing radiation acquires the characteristic forms of the polarization spectra. These spectra depend on the value and geometry of the magnetic field and on the distribution of the number density of electrons in a plasma. Analysis of these spectra can be useful for estimating the magnetic field and the electron concentration.

The polarization of the observed (the intensity is integrated over the surface of the atmosphere) radiation from the pointlike source reflected from a semi-infinite non-magnetized atmosphere was first calculated by Grinin \& Domke (1971). Following the ideas of Sobolev (1963), they obtained the integral matrix equation for the probability of the total energy of the polarized radiation escape from the semi-infinite atmosphere. The solution of this equation by the known Sobolev's method (see Sobolev 1968) has shown that the maximum polarization

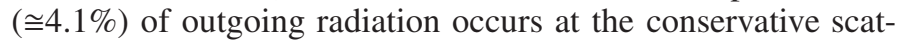
tering $(q=0)$ when the angle between the normal to the atmosphere $\boldsymbol{N}$ and the line of sight $\boldsymbol{n}$ is equal to $\cong 70^{\circ}$.

The influence of Faraday's rotation on the polarization plane in optically thick non-turbulent atmospheres has been studied in a number of papers: see Silant'ev (1979, 1994, 2002), Agol \& Blaes (1996), Agol et al. (1998), Shternin et al. (2003), etc.

\subsection{Faraday rotation in turbulent atmospheres}

The real atmospheres, accretion disks, and circumstellar envelopes are frequently turbulent. The frozen magnetic fields in these cases are also stochastic. Faraday rotation of the polarization plane, as a whole, diminishes the value of observed polarization. Besides, the spectra of polarization acquire new characteristic forms corresponding to the more rapid decrease in the polarization with the increase in the wavelengths. The theoretical calculations of the polarization spectra for various situations are very useful for estimating the magnetic fields and other parameters in many cosmic objects. Our paper is devoted to doing this.

The radiative transfer equation for the turbulent magnetized atmosphere was derived in a recent paper (Silant'ev 2005). In this atmosphere, all the quantities - the extinction coefficient $\alpha=\alpha_{0}+\alpha^{\prime}$, the magnetic field $\boldsymbol{B}=\boldsymbol{B}_{0}+\boldsymbol{B}^{\prime}$, the parameter of Faraday's rotation (see Sect. 2) $\delta=\delta_{0}+\delta^{\prime}$ and the Stokes parameters $I=I_{0}+I^{\prime}, Q=Q_{0}+Q^{\prime}, U=U_{0}+U^{\prime}$ and 
$V=V_{0}+V^{\prime}-$ are the stochastic quantities; i.e., they are characterized by its mean values (for example, $\langle I\rangle \equiv I_{0}$ ) and fluctuations (for example $I^{\prime},\left\langle I^{\prime}\right\rangle \equiv 0$ ). The brackets \langle\rangle denote the statistical average over the ensemble of the realizations of the stochastic quantity. Clearly (see Levshakov \& Kegel 1997) when we observe the radiation from many turbulent (more generally, stochastic) cells, we observe the mean values $I_{0}, Q_{0}, U_{0}$, and $V_{0}$. Silant'ev (2005) showed that if the characteristic radius $R_{0}$ of turbulent pulsations is less than the photon free path, i.e. $\tau_{1}=\alpha_{0} R_{0} \ll 1$ with $\tau_{1}$ being the mean optical depth of the turbulent cell, then the mean Stokes parameters obey the usual radiative transfer equation with renormalized (effective) kinetic coefficients.

Thus, instead of the extinction coefficient $\alpha$, we have to use the effective extinction factor $\alpha_{\text {eff }}$, which is less than the mean extinction coefficient $\alpha_{0}$. The difference between them is proportional to $\tau_{1} \ll 1$ and the level of fluctuations $\left\langle\alpha^{\prime 2}\right\rangle / \alpha_{0}^{2}$. In this paper we consider the case when the product of these values is low and one takes $\alpha_{\text {eff }} \cong \alpha_{0}$. It seems in interstellar medium the case can also occur where the level of extinction factor fluctuations is high and the effective extinction $\alpha_{\text {eff }}$ is considerably lower than the mean value $\alpha_{0}$. Below we consider the multiple light scattering in a semi-infinite atmosphere and use the notion of dimensionless optical depth $\tau=\alpha_{0} s$. The use of new optical depth $\alpha_{\text {eff }} s$ slightly changes the whole theory (see Silant'ev 2005). For the conservative atmosphere, without the true absorption of light, the results obtained below do not change.

The influence of the Faraday rotation fluctuations in a turbulent atmosphere can be very strong. Indeed, the fluctuating Faraday rotation angle $\psi_{1}^{\prime} \propto R_{0} \lambda^{2} B^{\prime}$ at the characteristic distance $R_{0}$ of the turbulent curls can be large $\left(\psi_{1}^{\prime} \gg 1\right)$ for a large enough magnetic field $B^{\prime}$ and wavelength $\lambda$. Statistically this gives rise to the decrease in the polarization degree of each light beam by its propagation in the turbulent magnetized medium. The effect is proportional to $\left\langle\left(\psi_{1}^{\prime}\right)^{2}\right\rangle$; i.e., it is proportional to $\lambda^{4}\left\langle\left(B^{\prime}\right)^{2}\right\rangle$. Physically this decrease appears as a consequence of the summation of the incoherent realizations with very different Faraday's rotation angles. Remember that the propagation of the light beam in an atmosphere with the regular magnetic field $\boldsymbol{B}_{0}$ does not change its degree of polarization, but only the value of the positional angle $\chi$. The outgoing radiation is depolarized in this case as the consequence of summing the beams going from different atmosphere's depths, each with its own value for the positional angle. All the statistical effects can be obtained qualitatively from the consideration of two realizations models (see Silant'ev 2007). Clearly these statistical effects distort the observed polarization spectra of outgoing radiation.

Below we calculate a number of the observed polarization spectra of the radiation from point-like sources reflected off in the optically thick turbulent, magnetized atmospheres. Note that the integrated intensities of radiation over the perpendicular coordinates $\rho$ and the azimuthal angles $\phi$ (see Fig. 1) obey the usual radiative transfer equation. This means that the observed integrated Stokes parameters are connected to the solutions of classical problems corresponding to unbounded radiation fluxes. Of course, this is true for any restricted sources of radiation in a homogeneous atmosphere.

\section{Basic equations}

In the vast domain of the wavelengths and the magnetic fields, the inequality $\omega_{B} / \omega \cong 0.93 \times 10^{-8} \lambda[\mu \mathrm{m}] B[\mathrm{G}] \ll 1, \omega_{B}=$ $e B / m_{\mathrm{e}} c$ takes place. In this case the scattering cross-section is equal to the Thomson value $\sigma_{\mathrm{T}}=6.65 \times 10^{-25} \mathrm{~cm}^{2}$. It means

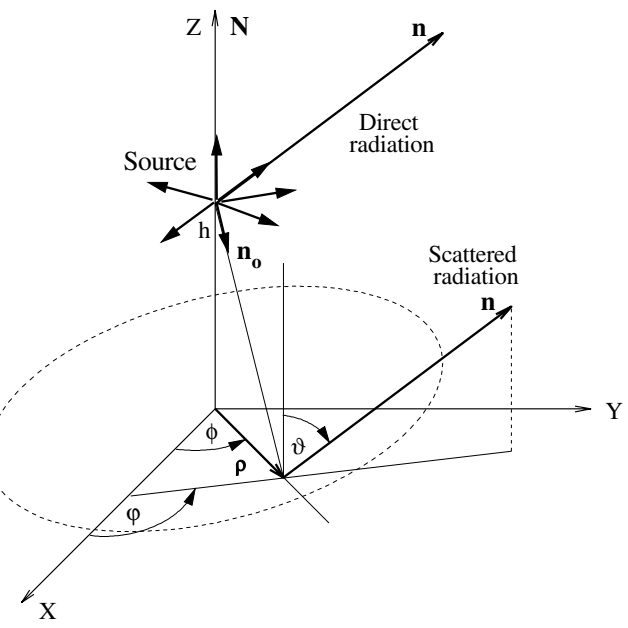

Fig. 1. Scheme of the multiple scattering of radiation from a pointlike source. The dashed curve denotes the characteristic dimension of a "spot".

that the magnetic field reveals itself only by the Faraday rotation of the polarization plane. The angle of Faraday's rotation $\psi$ can be written in the form (see Dolginov et al. 1995; Gnedin \& Silant'ev 1997)

$\psi=\frac{1}{2} \delta \tau_{\mathrm{T}} \cos \Theta$,

where $\tau_{\mathrm{T}}=N_{\mathrm{e}} \sigma_{\mathrm{T}} s$ is the Thomson optical length of the distance $s, N_{\mathrm{e}}$ the concentration of electrons in a plasma atmosphere, $\Theta$ the angle between magnetic field $\boldsymbol{B}$, and the light propagation direction (line of sight) $\boldsymbol{n}$. Remember that the polarization plane has a righthand rotation at $\Theta<90^{\circ}$ if we observe along the direction $\boldsymbol{n}$. At $\Theta>90^{\circ}$ the rotation is opposite. Parameter $\delta$ is determined by the formula

$\delta=\frac{3}{4 \pi} \frac{\lambda}{r_{\mathrm{e}}} \frac{\omega_{B}}{\omega} \cong 0.8(\lambda[\mu \mathrm{m}])^{2} B[\mathrm{G}]$.

Here $r_{\mathrm{e}}=e^{2} / m_{\mathrm{e}} c^{2} \cong 2.82 \times 10^{-13} \mathrm{~cm}$ is the classical radius of an electron, $\lambda=2 \pi c / \omega$ is the light wavelength, $\omega=2 \pi v$ the angular frequency, and $c$ the light velocity. Furthermore, we always take the wavelengths in $[\mu \mathrm{m}]$ and the magnetic fields in Gauss.

\subsection{Transfer equations for the average Stokes parameters}

According to the paper by Silant'ev (2005) the system of radiative transfer equations for the mean Stokes parameters in the turbulent magnetized atmosphere has the form

$$
\begin{aligned}
(\boldsymbol{n} \nabla) I_{0}(\boldsymbol{r}, \boldsymbol{n})= & -\alpha_{0} I_{0}(\boldsymbol{r}, \boldsymbol{n}) \\
& +\left\langle N_{\mathrm{e}} \sigma_{\mathrm{T}} B_{I}(\boldsymbol{r}, \boldsymbol{n})\right\rangle+\left\langle S_{I}(\boldsymbol{r}, \boldsymbol{n})\right\rangle, \\
(\boldsymbol{n} \nabla) Q_{0}(\boldsymbol{r}, \boldsymbol{n})= & -\alpha_{\mathrm{pol}} Q_{0}(\boldsymbol{r}, \boldsymbol{n}) \\
& -N_{0}(\boldsymbol{r}) \sigma_{\mathrm{T}} \delta_{\mathrm{eff}} \cos \Theta_{0} U_{0}(\boldsymbol{r}, \boldsymbol{n}) \\
& +\left\langle N_{\mathrm{e}} \sigma_{\mathrm{T}} B_{Q}(\boldsymbol{r}, \boldsymbol{n})\right\rangle+\left\langle S_{Q}(\boldsymbol{r}, \boldsymbol{n})\right\rangle, \\
(\boldsymbol{n} \nabla) U_{0}(\boldsymbol{r}, \boldsymbol{n})= & -\alpha_{\mathrm{pol}} U_{0}(\boldsymbol{r}, \boldsymbol{n}) \\
& +N_{0}(\boldsymbol{r}) \sigma_{\mathrm{T}} \delta_{\mathrm{eff}} \cos \Theta_{0} Q_{0}(\boldsymbol{r}, \boldsymbol{n}) \\
& +\left\langle N_{\mathrm{e}} \sigma_{\mathrm{T}} B_{U}(\boldsymbol{r}, \boldsymbol{n})\right\rangle+\left\langle S_{U}(\boldsymbol{r}, \boldsymbol{n})\right\rangle,
\end{aligned}
$$


where $N_{0}(\boldsymbol{r}) \equiv\left\langle N_{\mathrm{e}}(\boldsymbol{r})\right\rangle$ is the mean concentration of electrons, and $\Theta_{0}$ is the angle between the light propagation direction $\boldsymbol{n}$ and the mean magnetic field $\boldsymbol{B}_{0}$. The effective (renormalized) coefficients $\alpha_{\mathrm{pol}}$ and $\delta_{\text {eff }}$ take the following form:

$\alpha_{\mathrm{pol}}=\alpha_{0}+N_{0} \sigma_{\mathrm{T}} \tau_{1}^{(T)}\left[\left\langle\delta^{\prime 2}\right\rangle f_{B}+\delta_{0}^{2} \cos ^{2} \Theta_{0} \frac{\left\langle N_{\mathrm{e}}^{\prime 2}\right\rangle}{N_{0}^{2}} f_{N}\right]$,

$\delta_{\text {eff }}=\delta_{0}\left[1-2 \tau_{1} \frac{\left\langle\alpha^{\prime} N_{e}^{\prime}\right\rangle}{\alpha_{0} N_{0}} f_{\alpha N}\right]$

Here $\tau_{1}^{(\mathrm{T})}=N_{0} \sigma_{\mathrm{T}} R_{0}$ is the Thomson optical length of the characteristic radius of the turbulent correlations $R_{0}, \tau_{1}=\alpha_{0} R_{0}$ is the total optical depth of this radius, taking the true absorption into account. The $\delta_{0}=0.8 \lambda^{2} B_{0}$ is the mean value of the parameter $\delta$. The $\left\langle\left(\delta^{\prime}\right)^{2}\right\rangle=\left(0.8 \lambda^{2}\right)^{2}\left\langle\left(B^{\prime}\right)^{2}\right\rangle / 3$ is the rms-value of parameter $\delta^{\prime}$. The other averaged fluctuations have the same meaning. The values $f_{B}, f_{N}$, and $f_{\alpha N}$ are the integrals from the corresponding twopoint correlation functions (see Silant'ev 2005). All these factors are near unity. Frequently one uses the model of the normalized correlation function $\exp \left(-R / R_{0}\right)$. Here $R$ is the distance between the points where the fluctuating values are located. For this particular form of the correlation function, the coefficients $f_{B}, f_{N}$, and $f_{\alpha N}$ are equal to unity.

We see that the extinction coefficients of the intensities of polarized light (parameters $Q$ and $U$ ) acquire the additional terms due to the existence of the Faraday rotation fluctuations mentioned above. Note that these fluctuations can also be generated by the fluctuations of the electron number density (the second term in square brackets). Both terms in this brackets are proportional to $\lambda^{4}$ and can be greater at large $\lambda$ than the parameter $\delta_{0} \sim \lambda^{2}$. The second term in Eq. (5) describes the decrease in the positional angle in turbulent medium. When the regular magnetic field is absent $\left(\delta_{0}=0\right)$, this effect disappears since the fluctuating fields $\boldsymbol{B}^{\prime}$ and $-\boldsymbol{B}^{\prime}$ occur with equal frequency and the resulting average rotation of the position angle is equal to zero. This new effect is discussed in Silant'ev (2007) in more detail. This effect is practically small because is proportional to $\tau_{1}$. For this reason we take $\delta_{\text {eff }} \cong \delta_{0}$.

Our system (3) takes into account both the additional extinction of parameters $Q$ and $U$ and the usual decrease in the polarization degree due to the existence of the very different Faraday rotations of the polarization plane of light escaping from different layers of the atmosphere. Our particular calculations will be concerned with the case of a purely fluctuating magnetic field. This does not correspond to zero's mean magnetic field. Below we give the condition where the regular magnetic field $\boldsymbol{B}_{0}$ influences the light polarization negligibly less than the magnetic fluctuations. Of course, the other effects of the regular magnetic field can be considered.

Terms with $B_{I, Q, U}$ describe the Thomson scattering on the electrons. Their explicit forms are rather complex (see Chandrasekhar 1950). Therefore we use a more compact equation for the radiation density matrix $\rho_{\alpha \beta}(\boldsymbol{r}, \boldsymbol{n})$, which is linearly related to the Stokes parameters $I, Q, U, V$ (see Silant'ev 1979, 1994, 2005, 2007).

\subsection{Transfer equation for the radiation density matrix}

As is well known in plasma with $\omega_{B} / \omega \ll 1$, the electromagnetic waves with the right - and left - circular polarizations propagate independently each with its own refractive index. For this reason we use the most convenient representation of the radiation density matrix in cyclic coordinates (the unit vectors are $\left.\boldsymbol{e}_{-1}=\left(\boldsymbol{e}_{x}+\mathrm{i} \boldsymbol{e}_{y}\right) / \sqrt{2}, \boldsymbol{e}_{0}=\boldsymbol{e}_{z}, \boldsymbol{e}_{1}=-\left(\boldsymbol{e}_{x}-\mathrm{i} \boldsymbol{e}_{y}\right) / \sqrt{2}\right)$ :

$\rho_{-1-1}=\frac{1}{2}\left(I_{0}+V_{0}\right), \quad \rho_{11}=\frac{1}{2}\left(I_{0}-V_{0}\right)$,
$\rho_{-11}=\frac{1}{2}\left(-Q_{0}+\mathrm{i} U_{0}\right)$,

Here $V$ is the Stokes parameter describing the circularly polarized intensity. This parameter obeys the separate equation. We are interested in the linear polarization in this paper. Therefore we can take $V \equiv 0$. Instead of terms $B_{I, Q, U}$, we use the corresponding expressions in cyclic coordinates:

$\left\langle N_{\mathrm{e}} \sigma_{T} B_{\alpha \beta}(\boldsymbol{r}, \boldsymbol{n})\right\rangle=\alpha_{0} a_{n m}^{\alpha \beta}(\boldsymbol{n}) G_{n m}(\boldsymbol{r})$,

where we assume the summation over the repeated Greek $\alpha, \beta, \gamma, \ldots=-1,1$ and Latin $n, m, p, \ldots=-1,0,1$ indices, both here and in what follows. The angular distribution of the scattered radiation is described by the matrix

$a_{n m}^{\alpha \beta}(\boldsymbol{n})=\mathrm{e}^{-(n-m) \varphi} d_{n \alpha}^{(1)}(\vartheta) d_{m \beta}^{(1)}(\vartheta)$,

where the Wigner matrices (see Varshalovich et al. 1988) have the form

$d_{-1-1}^{(1)}=d_{11}^{(1)}=(1+\cos \vartheta) / 2, \quad d_{00}^{(1)}=\cos \vartheta$,

$d_{-11}^{(1)}=d_{1-1}^{(1)}=(1-\cos \vartheta) / 2$

$d_{01}^{(1)}=d_{-10}^{(1)}=-d_{10}^{(1)}=-d_{0-1}^{(1)}=\sin \vartheta / \sqrt{2}$.

The angles $\varphi$ and $\vartheta$ are the usual azimuthal and polar angles of the vector $\boldsymbol{n}$ in the reference frame with the $Z$-axis along the outward normal $N$ (see Fig. 1). The Stokes parameters $Q$ and $U$ are taken in the reference frame $\left(X_{n}, Y_{n}, Z_{n}\right)$ with the $Z_{n}$-axis in the direction $\boldsymbol{n}$. The $X_{n}$-axis is always taken in the plane $(\boldsymbol{n} \boldsymbol{N})$. Now the system of Eqs. (3) can be written as a one-matrix equation:

$$
\begin{aligned}
(\boldsymbol{n} \nabla) \rho_{\alpha \beta}(\boldsymbol{r}, \boldsymbol{n})= & -\alpha_{0} \bar{g}_{\alpha \beta}(\boldsymbol{n}) \rho_{\alpha \beta}(\boldsymbol{r}, \boldsymbol{n}) \\
& +\alpha_{0} a_{n m}^{\alpha \beta}(\boldsymbol{n}) G_{n m}(\boldsymbol{r})+\left\langle S_{\alpha \beta}(\boldsymbol{r}, \boldsymbol{n})\right\rangle .
\end{aligned}
$$

One of the advantages of using the radiation density matrix representation is that every matrix component propagates in the medium with its own absorption coefficient:

$\rho_{\alpha \beta}(s, \boldsymbol{n})=\rho_{\alpha \beta}(0, \boldsymbol{n}) \exp \left(-\alpha_{0} \bar{g}_{\alpha \beta} s\right)$,

where the dimensionless coefficients $\bar{g}_{\alpha \beta}(\boldsymbol{n})$ are determined by the relations

$\bar{g}_{-1-1}=\bar{g}_{11}=1$,

$\bar{g}_{-11}=\bar{g}_{1-1}^{*}=\left(\alpha_{\mathrm{pol}}+i N_{0} \sigma_{\mathrm{T}} \delta_{0} \cos \Theta_{0}\right) / \alpha_{0}$.

Note that there is no summation over repeated indices $\alpha$ and $\beta$ in Eqs. (10) and (11). Tensor $G_{n m}(\boldsymbol{r})$ is related to the density matrix (6):

$G_{n m}(\boldsymbol{r})=\frac{3}{8 \pi}(1-q) \int \mathrm{d} \boldsymbol{n}^{\prime} a_{n m}^{\gamma \nu *}\left(\boldsymbol{n}^{\prime}\right) \rho_{\gamma v}\left(\boldsymbol{r}, \boldsymbol{n}^{\prime}\right)$.

Here $1-q=N_{0} \sigma_{\mathrm{T}} / \alpha_{0}$, where $q$ is the degree of the true absorption. 


\subsection{Point-like source of radiation}

The terms $\left\langle S_{I}\right\rangle,\left\langle S_{Q}\right\rangle,\left\langle S_{U}\right\rangle$ and corresponding matrix $\left\langle S_{\alpha \beta}\right\rangle$ describe the averaged sources in the turbulent atmosphere. Furthermore, we consider the case where the point-like source of the anisotropic and polarized radiation is placed at height $h$ over the semi-infinite plane-parallel atmosphere (see Fig. 1). This source is described by the density matrix $L_{\gamma v}\left(\boldsymbol{n}_{0}\right)\left(\mathrm{erg} \mathrm{s}^{-1}\right.$ sterad $\left.^{-1}\right)$. The total luminosity of the source $L_{0}\left(\mathrm{erg} \mathrm{s}^{-1}\right)$ is equal to the integral of the value $L_{\gamma \gamma}\left(\boldsymbol{n}_{0}\right)$ over all solid angles $\mathrm{d} \boldsymbol{n}_{0}$. Note that, in contrast to the isotropic source, the anisotropic source can radiate the polarized radiation; for example, this is the dipole radiation. The density matrix of the radiation penetrating into the point $(-z, \rho, \phi)$ of the atmosphere (we use the cylindrical reference frame) is

$\rho_{\alpha \beta}^{(0)}\left(\boldsymbol{r}, \boldsymbol{n}^{\prime}\right)=L_{\alpha \beta}\left(\boldsymbol{n}_{0}\right) \frac{\exp \left(-\bar{g}_{\alpha \beta}\left(\boldsymbol{n}_{0}\right) \tau /\left|\mu_{0}\right|\right)}{(h+|z|)^{2}+\rho^{2}} \delta\left(\boldsymbol{n}^{\prime}-\boldsymbol{n}_{\mathbf{0}}\right)$,

where $\boldsymbol{n}_{0}$ is directed from the point-like source to the point $\boldsymbol{r}(-z, \rho, \phi)$. The $Z$-axis, as usual, is directed along the outward normal $N$ to the atmosphere. The source is located at $z=h$, and $\mu_{0}=\cos \vartheta_{0} \leq 0, \vartheta_{0}$ is the angle between $z$ and $\boldsymbol{n}_{0}$, and $\mathrm{d} \tau=-\alpha_{0} \mathrm{~d} z$, i.e. $\tau$ is the mean total optical depth of the level $-z$. Note that the azimuthal angles $\phi$ of the radius-vector $\boldsymbol{r}$ and the direction $\boldsymbol{n}_{0}$ coincide one with another.

Note also that the value $\mu_{0}$ is related to the coordinates $z$ and $\rho$ :

$$
\begin{aligned}
& \left|\mu_{0}\right|=\frac{h+|z|}{\sqrt{(h+|z|)^{2}+\rho^{2}}}, \\
& \left(\mathrm{~d} \mu_{0}\right)_{z=\text { const. }}=-\frac{\mu_{0}^{3} \rho \mathrm{d} \rho}{(h+|z|)^{2}} .
\end{aligned}
$$

The substitution of matrix (14) in formula (13) gives rise to the matrix $G_{n m}^{(0)}(\boldsymbol{r})$ :

$G_{n m}^{(0)}(\boldsymbol{r})=\frac{3}{8 \pi}(1-q) a_{n m}^{\gamma \nu *}\left(\boldsymbol{n}_{0}\right) L_{\gamma \nu}\left(\boldsymbol{n}_{0}\right) \frac{\exp \left(-\bar{g}_{\gamma \nu}\left(\boldsymbol{n}_{0}\right) \tau /\left|\mu_{0}\right|\right)}{(h+|z|)^{2}+\rho^{2}}$.

Note that, due to relation (15), this matrix depends on the height $h$. The substitution of this matrix to Eq. (7) gives the explicit form of the source $\left\langle S_{\alpha \beta}(\boldsymbol{r}, \boldsymbol{n})\right\rangle$. Remember that source functions $\left\langle S_{I, Q, U}\right\rangle$ and corresponding $\left\langle S_{\alpha \beta}\right\rangle$ are equal to the scattering term (7) after the substitution the intensities of radiation penetrating the atmosphere (see expression (14)).

\subsection{Formulae for the observed integral polarization}

The atmosphere is considered as homogeneous in directions perpendicular to the $Z$-axis. The dependence of the Stokes parameters $I, Q, U$ from the perpendicular distance $\rho$ and the azimuthal angle $\phi$ is due to the inhomogeneity and anisotropy of the source (see Eq. (17)). The derivatives along the direction $\boldsymbol{n}$ on the lefthand sides of the systems (3) and (10) also have the derivatives over $\rho$ and $\phi$. For this reason the solution of equations for $I(\tau, \rho, \phi, \boldsymbol{n}), Q(\tau, \rho, \phi, \boldsymbol{n})$, and $U(\tau, \rho, \phi, \boldsymbol{n})$ is a very difficult problem.

From expressions (14) and (17), we see that the intensity of the radiation penetrating the medium decreases rapidly in the perpendicular directions; i.e., it has the spot-like distribution with the characteristic length $\rho_{*} \geq h$. The telescope observe, the "spot" as a whole. If the curvature radius of the atmosphere is large compared with the characteristic dimension of the "spot", we can consider the atmosphere as a plane with inclination angle $\vartheta$. The angle $\vartheta$ is the angle between the line of sight $\boldsymbol{n}$ and the local outward normal to the atmosphere $N(\mu \equiv \cos \vartheta)$. In this case the telescope registers the radiation fluxes $F_{I}(\boldsymbol{n}), F_{Q}(\boldsymbol{n})$, and $F_{U}(n)$ proportional to $\mu$ and the surface integrals over the "spot":

$$
\begin{aligned}
& F_{I}(\boldsymbol{n})=\frac{\mu}{R^{2}} \int_{0}^{2 \pi} \mathrm{d} \phi \int_{0}^{\infty} \mathrm{d} \rho \rho I_{0}(\boldsymbol{r}, \boldsymbol{n}) \equiv \frac{\mu}{R^{2}} E_{I}(\boldsymbol{n}), \\
& F_{Q}(\boldsymbol{n})=\frac{\mu}{R^{2}} \int_{0}^{2 \pi} \mathrm{d} \phi \int_{0}^{\infty} \mathrm{d} \rho \rho Q_{0}(\boldsymbol{r}, \boldsymbol{n}) \equiv \frac{\mu}{R^{2}} E_{Q}(\boldsymbol{n}), \\
& F_{U}(\boldsymbol{n})=\frac{\mu}{R^{2}} \int_{0}^{2 \pi} \mathrm{d} \phi \int_{0}^{\infty} \mathrm{d} \rho \rho U_{0}(\boldsymbol{r}, \boldsymbol{n}) \equiv \frac{\mu}{R^{2}} E_{U}(\boldsymbol{n}),
\end{aligned}
$$

where $R$ is the distance to the telescope, and the values $E_{I, Q, U}(\boldsymbol{n})$ are the surface integrals from $I_{0}(0, \rho, \phi, \boldsymbol{n}), Q_{0}(0, \rho, \phi, \boldsymbol{n})$, and $U_{0}(0, \rho, \phi, \boldsymbol{n})$. These values have the meaning of total energy $\left(\mathrm{erg} \mathrm{s}^{-1}\right.$ sterad $\left.^{-1}\right)$, radiating from the whole "spot" to the direction $\boldsymbol{n}$ (see Fig. 1).

Clearly at $\rho \rightarrow \infty$ the intensity tends to zero. Therefore by integrating Eqs. (3) or (10) over surfaces $\tau=$ const., terms with the derivatives over $\rho$ and $\phi$ do not give any contribution. Thus, the values $E_{I, Q, U}(\tau, \boldsymbol{n})$ obey the usual transfer equations without the derivatives over $\rho$ and $\phi$. The source terms in this system of equations are equal to the source terms in Eq. (3) integrated over the variables $\rho$ and $\phi$. It seems that in first time this was mentioned by Sobolev (1963) and later used by Grinin and Domke (1971), when derivation of the system of equations for the polarized radiation probabilities to escape the non-turbulent and non-magnetized atmosphere.

If we consider the bounded flux of the plane-parallel light, then the expressions for $E_{I, Q, U}$ coincide with the known solution for the unbounded flux and need to be multiplied on the surface area of the "spot" $S_{0} /\left|\cos \vartheta_{0}\right|$. Here $S_{0}$ is the cross-section of the beam and $\vartheta_{0}$ is its inclination angle.

\subsection{Relation to the known solutions for unbounded beams}

The solution methods for the usual system of equations for the Stokes parameters are known. Silant'ev (1994) developed the generalization of the Sobolev (1968) method to solve a number of the standard problems, in particular, the problem of reflecting the parallel unbounded polarized light beam off in the semiinfinite non-turbulent magnetized atmosphere. The problem of the reflection of the polarized light from a magnetized atmosphere was also resolved earlier (Silant'ev 1979) using Eq. (10) and the known invariance principle (Chandrasekhar 1950).

Below we calculate the directly observed radiation fluxes (18); i.e., we have to solve the usual radiative transfer equations for the values $E_{I, Q, U}(\tau, \boldsymbol{n})$. For this we must obtain the surface integrals over planes $\tau=$ const. from the function $G_{n m}^{(0)}(\boldsymbol{r})$. According to expression (16), this surface integration can be transformed into the solid angles integration over the semi-sphere of the solid angles $\Omega^{-}$directed into the interior of the atmosphere:

$$
\begin{aligned}
\int_{0}^{\infty} \mathrm{d} \rho \rho & \int_{0}^{2 \pi} \mathrm{d} \phi G_{n m}^{(0)}(\boldsymbol{r})=\frac{3}{8 \pi}(1-q) \\
& \times \int_{\Omega^{-}} \frac{\mathrm{d} \boldsymbol{n}_{0}}{\left|\mu_{0}\right|} a_{n m}^{\gamma \nu *}\left(\boldsymbol{n}_{0}\right) L_{\gamma \nu}\left(\boldsymbol{n}_{0}\right) \exp \left(-\bar{g}_{\gamma \nu}\left(\boldsymbol{n}_{0}\right) \tau /\left|\mu_{0}\right|\right) .
\end{aligned}
$$


Note that, after this integration, the problem does not depend on the altitude $h$ of the point-like source over atmosphere's surface. This independence is entirely the consequence of the relations (16) taking place only for point-like sources, both the isotropic and anisotropic. It means that any distribution of these sources along the normal $\boldsymbol{N}$ will be observed as an effective point-like source. We are really restricted by the condition that the effective radius of "spot" $\left(\rho_{*} \geq h\right)$ has to be smaller than the curvature radius of the atmosphere (see Sect. 2.4). As a result, very high values of $h$ are beyond the validity of this paper. In this subsection we demonstrate that the observed radiation fluxes (18) can be calculated from the known solution to the problem of the reflections unbounded plane-parallel beam from semi-infinite plane-parallel atmosphere. The continued source producing the "spot" smaller than the curvature of the atmosphere can be considered as a sum of point-like sources, so our consideration includes these more realistic forms of sources. It is important that the inclination angle of the "spot" is practically the same for all the parts of the "spot".

Comparison of expression (19) with formula (44) in the paper (Silant'ev 1994) or with formula (12) in Silant'ev (1979) shows that this expression is the integral superposition of the sources for the known classical problem on the reflection of unbounded light beam from the semi-infinite non-turbulent magnetized medium. If the density matrix $F_{\gamma \nu}^{(0)}\left(\boldsymbol{n}_{0}\right)$ in formula (44) is replaced by $L_{\gamma \nu}\left(\boldsymbol{n}_{0}\right) /\left|\mu_{0}\right|$, then we obtain the integrand in expression (19). Note that the notations in Silant'ev (1979) differ from those in Silant'ev (1994).

The solution of this classical problem (see formulae (45) and (12) in the mentioned papers) takes the form:

$$
\begin{aligned}
\rho_{\alpha \beta}\left(\boldsymbol{n}, \boldsymbol{n}_{0}, \boldsymbol{B}_{0}\right)= & \frac{3}{8 \pi}(1-q)\left|\mu_{0}\right|(-1)^{n+m} \\
& \times \frac{C_{n m}^{\alpha \beta}\left(\boldsymbol{n}, \boldsymbol{B}_{0}\right) C_{-n-m}^{\gamma v}\left(-\boldsymbol{n}_{0},-\boldsymbol{B}_{0}\right) F_{\gamma v}^{(0)}\left(\boldsymbol{n}_{0}\right)}{\mu \bar{g}_{\gamma v}\left(\boldsymbol{n}_{0}, \boldsymbol{B}_{0}\right)+\left|\mu_{0}\right| \bar{g}_{\alpha \beta}\left(\boldsymbol{n}, \boldsymbol{B}_{0}\right)} .
\end{aligned}
$$

Here, $F_{\gamma \nu}^{(0)}\left(\boldsymbol{n}_{0}\right)$ and $\rho_{\alpha \beta}\left(\boldsymbol{n}, \boldsymbol{n}_{0}, \boldsymbol{B}_{0}\right)$ are the density matrices of the radiative flux falling along the direction $\boldsymbol{n}_{0}$ and the radiation reflected in direction $\boldsymbol{n}$, and $\boldsymbol{B}_{0}$ is a homogeneous magnetic field in the atmosphere. Note that the coefficients $g_{\alpha \beta}$ in these formulae are to be replaced by our coefficients (12), which include the additional extinction factor $\alpha_{\text {pol }}$. After these changes, the formulae (45) and (12) present the solution of the classical problem in the turbulent magnetized atmosphere with $\boldsymbol{B}_{0}$ as the mean value of the magnetic field. The magnetic field fluctuations are presented in the coefficients (12)

The tensor functions $C_{n m}^{\alpha \beta}\left(\boldsymbol{n}, \boldsymbol{B}_{0}\right)$ are the generalization of known Ambartzumyan - Chandrasekhar $\mathrm{H}$ - functions (see Ambartzumyan 1942; Chandrasekhar 1950). They obey the following system of nonlinear equations (Silant'ev 1979, 1994):

$$
\begin{aligned}
C_{p q}^{\alpha \beta}\left(\boldsymbol{n}, \boldsymbol{B}_{0}\right)= & a_{p q}^{\alpha \beta}(\boldsymbol{n})+\frac{3}{8 \pi}(1-q) \mu \\
& \times \int_{\Omega^{+}} \mathrm{d} \boldsymbol{n}^{\prime} \frac{C_{n m}^{\alpha \beta}\left(\boldsymbol{n}, \boldsymbol{B}_{0}\right) C_{n m}^{\gamma v *}\left(\boldsymbol{n}^{\prime},-\boldsymbol{B}_{0}\right) a_{p q}^{\gamma v}\left(\boldsymbol{n}^{\prime}\right)}{\bar{g}_{\gamma v}\left(\boldsymbol{n}^{\prime}, \boldsymbol{B}_{0}\right) \mu+\bar{g}_{\alpha \beta}\left(\boldsymbol{n}, \boldsymbol{B}_{0}\right) \mu^{\prime}} .
\end{aligned}
$$

The solid angle region $\Omega^{+}$of the integration corresponds to values with $\mu^{\prime} \geq 0$. The number of independent components of the tensor $C_{n m}^{\alpha \beta}$ is reduced substantially by the large number of symmetry properties; in particular, the matrix is hermitian, $C_{n m}^{\alpha \beta}=C_{m n}^{\beta \alpha *}$. For axially symmetric problems with the magnetic field directed along the normal to the atmosphere the system (21) break up a number of separate systems. The maximum order of these systems is equal to 6 (for $n-m=0$ ).

Note that Eqs. (20) and (21) can be easily derived from radiative transfer Eq. (10) using the standard procedure of Chandrasekhar (1950) as in Silant'ev (1979). In this paper the value $H_{p q}^{\alpha \beta} \equiv C_{p q}^{\alpha \beta}$. Note also that the property $(-1)^{p+q} H_{-p-q}^{-\gamma-\gamma}=$ $C_{p q}^{\gamma \nu *}$ occurs.

Due to the linearity of the transfer Eqs. (3) and (10), the problem of the reflection of the total energies $E_{I, Q, U}$ from the semi-infinite medium will be expressed as the integral over the semi-sphere of solid angles $\Omega^{-}$corresponding to all the directions $\boldsymbol{n}_{0}$ from the expression (20) where instead of $F_{\gamma \nu}^{(0)}\left(\boldsymbol{n}_{0}\right)$ we substitute the value $L_{\gamma \nu}\left(\boldsymbol{n}_{0}\right) /\left|\mu_{0}\right|$.

Thus, the general formula for the density matrix of the multiple scattered radiation flux registered by the telescope takes the form

$F_{\alpha \beta}\left(\boldsymbol{n}, \boldsymbol{B}_{0}\right)=\frac{3(1-q)}{8 \pi} \frac{\mu}{R^{2}}(-1)^{n+m}$

$\times \int_{\Omega^{-}} \mathrm{d} \boldsymbol{n}_{0} \frac{C_{n m}^{\alpha \beta}\left(\boldsymbol{n}, \boldsymbol{B}_{0}\right) C_{-n-m}^{\gamma v}\left(-\boldsymbol{n}_{0},-\boldsymbol{B}_{0}\right) L_{\gamma v}\left(\boldsymbol{n}_{0}\right)}{\mu \bar{g}_{\gamma v}\left(\boldsymbol{n}_{0}, \boldsymbol{B}_{0}\right)+\left|\mu_{0}\right| \bar{g}_{\alpha \beta}\left(\boldsymbol{n}, \boldsymbol{B}_{0}\right)}$.

Formulae for the Stokes parameters $F_{I}, F_{Q}$, and $F_{U}$ can be obtained from this expression if one uses the relations (6). In particular, this general solution represents the isotropic atmosphere with $\boldsymbol{B}_{0}=0$ and isotropic magnetic fluctuations.

The expressions for the parameters $F_{I, Q, U}$ determine the total radiation reflected by the atmosphere after the multiple scattering. Because these formulae do not depend on altitude $h$, each distribution of sources can be considered as an effective source with the luminosity $L_{\gamma v}^{\text {eff }}\left(\boldsymbol{n}_{0}\right)=L_{\gamma \nu}^{(1)}\left(\boldsymbol{n}_{0}\right)+L_{\gamma \nu}^{(2)}\left(\boldsymbol{n}_{0}\right)+\ldots$ The telescope also registers the radiation directly coming from the sources. It means that we are adding the value $L_{\gamma v}^{\mathrm{eff}}(\boldsymbol{n}) / R^{2}$ to expression (22). Remember that we are restricted by the condition that the radius of the atmosphere curvature is larger than the characteristic surface dimension of the radiating "spot".

As mentioned above, transfer equations (3) for the total energies $E_{I, Q, U}$ take place for any forms of bounded sources, not only for the point-like sources, for example, for the bounded beam of polarized radiation falling in the direction $\boldsymbol{n}_{0}$. In the case of $F_{\alpha \beta}\left(\boldsymbol{n}, \boldsymbol{B}_{0}\right)$, we obtain the expression (22) where the integration over $\mathrm{d} \boldsymbol{n}_{0}$ is absent and the whole formula is multiplied by the factor $S_{0} /\left|\mu_{0}\right|$. Analogously, using the general theory of the paper (Silant'ev 1994), one can obtain the formulae for $F_{\alpha \beta}$ for other forms of spot-like sources, in particular, for spot-like sources of the thermal radiation inside the atmosphere.

\section{Reflection of radiation from the turbulent atmosphere with isotropic magnetic field fluctuations}

A turbulent atmosphere with isotropic magnetic field fluctuations is the simplest example of the influence of the magnetic field on the polarization of outgoing radiation. In Silant'ev (2007) devoted to Milne's problem in magnetized conservative atmospheres, it was shown that, if the inequality

$\tau_{1}^{(T)} \lambda^{2}\left\langle B^{\prime 2}\right\rangle \geq 4 B_{0}$

takes place, the influence of regular magnetic field $\boldsymbol{B}_{0}$ on the light polarization is weaker than the influence of magnetic fluctuations $\left\langle B^{\prime 2}\right\rangle$. In expression (23) the value $\tau_{1}^{(\mathrm{T})}$ is the Thomson optical depth of the characteristic length $R_{0}$ of turbulent pulsations. Remember that in this paper we use the wavelengths $\lambda$ in 
$[\mu \mathrm{m}]$, and the magnetic field in Gauss. It follows from inequality (23) that, at large wavelengths and (or) large magnetic field fluctuations, we can use the results of this section to the interpret observing polarization spectra even if the small regular magnetic field exists. This small field barely changes the polarization spectra.

The atmosphere with isotropic magnetic field fluctuations is isotropic. For this reason we can only consider the functions $C_{n m}^{\alpha \beta}(\boldsymbol{n})$ with $n=m$. Besides, in this case we can omit the explicit notions of the magnetic field in formulae. Indeed, the regular magnetic field $\boldsymbol{B}_{0}$ is neglected and the dependence on the scalar parameter $\left\langle B^{\prime 2}\right\rangle$ exists only in expressions (4) and (12), so the formulae for dimensionless parameters $\bar{g}_{\gamma v}$ in Eq. (12) acquire very simple form

$\bar{g}_{-1-1}=\bar{g}_{11}=1, \quad \bar{g}_{-11}=\bar{g}_{1-1}=1+(1-q) b \equiv g$,

$b=0.217 f_{B} \tau_{1}^{(\mathrm{T})} \lambda^{4}\left\langle B^{\prime 2}\right\rangle, \quad \tau_{1}^{(\mathrm{T})}=N_{0} \sigma_{\mathrm{T}} R_{0}$.

Parameter $b$ describes the additional decrease in the polarized part of the radiation due to the existence of incoherent Faraday rotation fluctuations of the polarization plane. At $b=0$ we return to the case of the usual non-magnetized electron atmosphere described by the Rayleigh phase matrix. This case was considered in Grinin \& Domke (1971).

Consideration of system (21) gives rise to following expressions for the functions $C_{n n}^{\alpha \beta}$ :

$C_{-1-1}^{\alpha \alpha}=C_{11}^{\alpha \alpha} \equiv A(\mu)$,

$C_{-1-1}^{\alpha-\alpha}=C_{11}^{\alpha-\alpha} \equiv C(\mu)$

$C_{00}^{\alpha \alpha} \equiv B(\mu), \quad C_{00}^{\alpha-\alpha} \equiv-D(\mu)$.

Here we take the condition $V \equiv 0$ into account. More general expressions without this condition and with the existence of the regular magnetic field directed along the normal $N$ are given in Silant'ev (2007).

The functions $A(\mu), B(\mu), C(\mu)$, and $D(\mu)$ obey the following system of nonlinear equations:

$$
\begin{aligned}
A(\mu)=\frac{1+\mu^{2}}{4}+\frac{3}{8}(1-q) \mu \\
\quad \times \int_{0}^{1} \mathrm{~d} x\left[\left(1+x^{2}\right) \frac{2 A(\mu) A(x)+B(\mu) B(x)}{\mu+x}\right. \\
\left.+\left(1-x^{2}\right) \frac{2 A(\mu) C(x)-B(\mu) D(x)}{g \mu+x}\right], \\
B(\mu)=\frac{1-\mu^{2}}{2}+\frac{3}{4}(1-q) \mu \\
\quad \times \int_{0}^{1} \mathrm{~d} x\left(1-x^{2}\right)\left[\frac{2 A(\mu) A(x)+B(\mu) B(x)}{\mu+x}\right. \\
\left.\quad-\frac{2 A(\mu) C(x)-B(\mu) D(x)}{g \mu+x}\right],
\end{aligned}
$$

$$
\begin{aligned}
C(\mu)= & \frac{1-\mu^{2}}{4}+\frac{3}{8}(1-q) \mu \\
& \times \int_{0}^{1} \mathrm{~d} x\left[\left(1+x^{2}\right) \frac{2 C(\mu) A(x)-D(\mu) B(x)}{\mu+g x}\right. \\
& \left.+\left(1-x^{2}\right) \frac{2 C(\mu) C(x)+D(\mu) D(x)}{g \mu+g x}\right], \\
D(\mu)= & \frac{1-\mu^{2}}{2}-\frac{3}{4}(1-q) \mu \\
& \times \int_{0}^{1} \mathrm{~d} x\left(1-x^{2}\right)\left[\frac{2 C(\mu) A(x)-D(\mu) B(x)}{\mu+g x}\right. \\
& \left.-\frac{2 C(\mu) C(x)+D(\mu) D(x)}{g \mu+g x}\right] .
\end{aligned}
$$

From this system we can derive the relations

$$
\begin{aligned}
2 A(\mu)+B(\mu) & =1 \\
+ & \frac{3}{2}(1-q) \mu \int_{0}^{1} \mathrm{~d} x \frac{2 A(\mu) A(x)+B(\mu) B(x)}{\mu+x} \\
2 C(\mu)-D(\mu) & =\frac{3}{2}(1-q) \mu \\
& \times \int_{0}^{1} \mathrm{~d} x \frac{2 C(\mu) A(x)-D(\mu) B(x)}{\mu+g x}
\end{aligned}
$$

Integration of the first relation gives rise to the connection between the zero moments $A_{0}$ and $B_{0}$ :

$$
\begin{aligned}
& 2 A_{0}+B_{0}=1+\frac{3}{4}(1-q)\left(2 A_{0}^{2}+B_{0}^{2}\right) \\
& A_{n}=\int_{0}^{1} \mathrm{~d} \mu \mu^{n} A(\mu), \quad B_{n}=\int_{0}^{1} \mathrm{~d} \mu \mu^{n} B(\mu) .
\end{aligned}
$$

Considering this relation as a quadratic equation for one of the moments, e.g., for $A_{0}$, we find that at $q=0$ the unique real solution is $A_{0}=B_{0}=2 / 3$.

\subsection{Effective methods of solution of system (26)}

The solution of the system (26) by a direct iteration method at small absorption, $q \ll 1$, is very ineffective, especially at $q=0$. Silant'ev (2007) found the method to improve the convergence of the iterations for $q=0$. To some extent this method is analogous to the known Chandrasekhar's method (see Chandrasekhar 1950). Remember that it is applied to solve the scalar nonlinear equations for $\mathrm{H}$-functions. Below we present the generalization of the Silant'ev (2007) method for any values of the absorption degree $q$.

Substituting the equality $\mu /(\mu+x)=1-x /(\mu+x)$ into the first relation in Eq. (27), we obtain the formula:

$$
A(\mu)=\frac{1-\frac{3}{2}(1-q) \int_{0}^{1} \mathrm{~d} x \frac{x B(\mu) B(x)}{\mu+x}-B(\mu)\left[1-\frac{3}{2}(1-q) B_{0}\right]}{3(1-q) \int_{0}^{1} \mathrm{~d} x \frac{x A(x)}{\mu+x}+\left[2-3(1-q) A_{0}\right]}
$$

At $q=0\left(A_{0}=2 / 3, B_{0}=2 / 3\right)$, the terms in the square brackets are equal to zero and we obtain the iteration formula for $A(\mu)$, used in the paper (Silant'ev 2007). It is seen that substitution 
of the approximate value of $A(\mu)$ with the underestimation gives rise to the value $A(\mu)$ with the overestimation; i.e., this is the fork method. The iterations of functions $B(\mu), C(\mu)$, and $D(\mu)$ are calculated directly from system (26). As a new approximation,one uses the half of sum of two previous approximations.

At $q \neq 0$ for the iterations of $A(\mu)$, we use formula (29), where the moments $A_{0}$ and $B_{0}$ correspond to the previous iteration, and the initial approximation coincides with the free terms in system (26). The initial values of the moments $A_{0}$ and $B_{0}$ are equal to $1 / 3$ and for every iteration these moments are calculated from the current iterations of $A(\mu)$ and $B(\mu)$. As a new approximation is also used, the half of sum of two previous approximations. Such procedure gives the quick calculation of $A, B, C$, and $D$ up to $q \simeq 0.001$. For the lowest $q$, this procedure is rather ineffective but still more effective than direct iterations of system (26). It should be noted that the function $A(\mu)$ grows monotonic from the value 0.25 at $\mu=0$, whereas the other functions first slightly increase with the increase in $\mu$ but later decrease monotonically (see Fig. 2). The functions $C(\mu)$ and $D(\mu)$ tend to zero at $\mu \rightarrow 1$. It seems that the fork method for $A(\mu)$, which is to some extent a maximal function compared with the other functions provides the effective convergence for the other $B(\mu), C(\mu)$, and $D(\mu)$ functions.

\subsection{General formulae for observed intensity and polarization}

Now we write the explicit formulae for the Stokes parameters $F_{I}(\mu)$ and $F_{Q}(\mu)$ (the parameter $F_{U} \equiv 0$ ) which follow from Eqs. (22) and (25):

$$
\begin{aligned}
F_{I}(\mu)= & \frac{1}{R^{2}} \frac{3}{2}(1-q) \mu \\
& \times \int_{0}^{1} \mathrm{~d} x\left[L_{I}(-x) \frac{2 A(\mu) A(x)+B(\mu) B(x)}{\mu+x}\right. \\
& \left.-L_{Q}(-x) \frac{2 A(\mu) C(x)-B(\mu) D(x)}{x+\mu g}\right], \\
F_{Q}(\mu)= & -\frac{3}{R^{2}} \frac{3}{2}(1-q) \mu \\
& \times \int_{0}^{1} \mathrm{~d} x\left[L(-x) \frac{2 C(\mu) A(x)-D(\mu) B(x)}{\mu+x g}\right. \\
& \left.-L_{Q}(-x) \frac{2 C(\mu) C(x)+D(\mu) D(x)}{x g+\mu g}\right] .
\end{aligned}
$$

Here we assume that the point-like source of the polarized radiation is axially symmetric:

$$
\begin{aligned}
& L_{-1-1}=L_{11}=L_{I}\left(\mu_{0}\right) / 2, \\
& L_{-11}=L_{1-1}^{*}=\left(-L_{Q}\left(\mu_{0}\right)+i L_{U}\left(\mu_{0}\right)\right) / 2 .
\end{aligned}
$$

If one takes $L_{\gamma v}\left(\boldsymbol{n}_{0}\right)$ depending from the azimuthal angle $\varphi_{0}$, then it is necessary also to calculate the functions $C_{n m}^{\alpha \beta}$ with $n \neq m$. Note that, in the axially symmetric case, the parameter $L_{U}$ does not give the contribution.

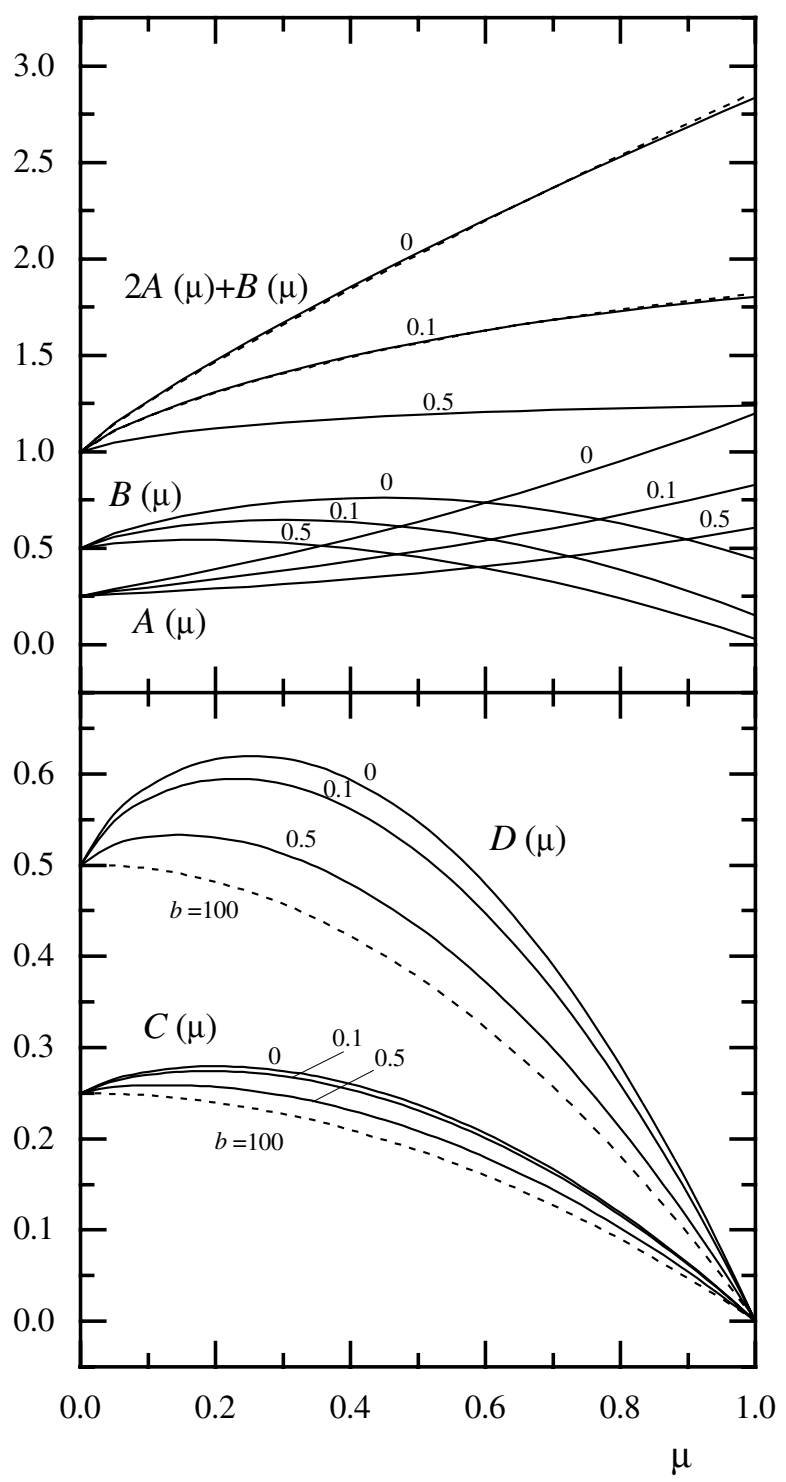

Fig. 2. The functions $A(\mu), B(\mu), C(\mu), D(\mu)$, and $2 A(\mu)+B(\mu)$ at $q=$ $0,0.1,0.5$, and $b=0$. The dashed curves for $C(\mu)$ and $D(\mu)$ corresponding to $b=100$ practically coincide for all the values of parameter $q$. The dashed curves for $2 A(\mu)+B(\mu)$ correspond to $b=100$.

\subsection{The case of an isotropic non-polarized source}

For this most simple type of source, we have $L_{\alpha \beta}=\delta_{\alpha \beta} L_{0} / 8 \pi$. From formula (30), together with relations (27), the very simple expressions follow (remember that $L_{0}$ is the total luminosity of the source):

$$
\begin{aligned}
& F_{I}(\mu)=\frac{L_{0}}{4 \pi R^{2}}(2 A(\mu)+B(\mu)-1), \\
& F_{Q}(\mu)=-\frac{L_{0}}{4 \pi R^{2}}(2 C(\mu)-D(\mu)) .
\end{aligned}
$$

The parameters $F_{I}$ and $F_{Q}$ have the dimension of the radiation flux $\left(\mathrm{erg} \mathrm{s}^{-1} \mathrm{~cm}^{-2}\right)$.

Taking into account the radiation emerging to the telescope directly from the source $L_{0} / 4 \pi R^{2}$ into account, for observing flux $F_{I}(\mu)$ we obtain the expression:

$F_{I}(\mu)=\frac{L_{0}}{4 \pi R^{2}}(2 A(\mu)+B(\mu))$ 
Thus, the actually observed polarization degree is

$p(\mu)=\frac{|2 C(\mu)-D(\mu)|}{2 A(\mu)+B(\mu)}$.

Note that the value $2 C-D \leq 0$ in all the calculations, i.e. the electric field oscillations tend occur in the plane $(n N)$, containing the line of sight $\boldsymbol{n}$ and the outward normal $\boldsymbol{N}$ to the atmosphere (this is the - so called negative polarization). It seems this is the general statement, though we did not prove this directly from system (26). In the classical Milne's problem (see Chandrasekhar 1950) that corresponds to diffusion of the thermal radiation from very deep layers of the atmosphere the polarization is positive, i.e. the polarization plane lies perpendicular to the plane $(n N)$. In this classical problem the radiation density gradient only has a vertical component.

In our case of the spot-like source, there is also the transverse gradient of the radiation density. Only this gradient is acceptable for the negative polarization. The qualitative explanation of this effect is the following. For the non-polarized radiation propagating inside the medium perpendicular to the plane $(\boldsymbol{n N})$, the scattering angle always is equal to $90^{\circ}$; i.e., the radiation scattered in the $\boldsymbol{n}$-direction is $100 \%$ polarized in the plane $(\boldsymbol{n N})$. In contrast, for the radiation propagating parallel to $(n N)$-plane the scattering angles mostly does not equal $90^{\circ}$, and the scattered radiation with the polarization perpendicular to the plane $(\boldsymbol{n N})$ is not $100 \%$ polarized. For this reason,the summarized radiation is negatively polarized. Of course, due to the axial symmetry of the problem, the polarization $p(\boldsymbol{n})$ is absent for $\boldsymbol{n} \| \boldsymbol{N}$. A more detailed explanation of the generation of the negative polarization is given in Dolginov et al. (1995).

Let us consider another simple source of point-like polarized radiation, the electric dipole with the axis along the normal $N$. Here one has $L_{I}=L_{Q}=\left(3 L_{0} / 8 \pi\right)\left(1-\mu_{0}^{2}\right), L_{U}=0$. The general formulae (30) and (26) give the following results:

$$
\begin{aligned}
& F_{I}(\mu)=\frac{3 L_{0}}{4 \pi R^{2}}\left[B(\mu)-\frac{1-\mu^{2}}{2}\right], \\
& F_{Q}(\mu)=-\frac{3 L_{0}}{4 \pi R^{2}}\left[D(\mu)-\frac{1-\mu^{2}}{2}\right] .
\end{aligned}
$$

Taking the direct radiation from the source into account, we derive the formulae

$$
\begin{aligned}
& F_{I}(\mu)=\frac{3 L_{0}}{4 \pi R^{2}} B(\mu), \\
& F_{Q}(\mu)=-\frac{3 L_{0}}{4 \pi R^{2}}\left[D(\mu)-\left(1-\mu^{2}\right)\right] .
\end{aligned}
$$

\subsection{The calculation of albedo}

As the conclusion of this section we derive the formulae for the albedo $a$ of the atmosphere, i.e. the ratio of the outgoing radiation energy to the total radiation energy penetrating into the medium from the source. For the case of an isotropic nonpolarized source, we have $a=2 A_{0}+B_{0}-1, a=3\left(B_{0}-1 / 3\right)$ for the dipole source. For the conservative atmosphere $\left(A_{0}=B_{0}=2 / 3\right)$, we obtain the value $a=1$, and for the fully absorbing atmosphere $\left(q=1, A_{0}=B_{0}=1 / 3\right)$, we obtain the value $a=0$. For $q=0.1,0.2$, and 0.5 , the isotropic source characterized by albedos equal to $0.519,0.382$, and 0.172 , respectively. It is interesting that the albedo does not depend on the value of magnetic fluctuations, because the $(2 A(\mu)+B(\mu))$ - function practically does not depend on the magnetic fluctuations (see also the next chapter). In the general case the integration of $F_{I}$ in Eq. (30) and the use of relations (27) give rise to the formula

$$
\begin{aligned}
a= & \int_{0}^{1} \mathrm{~d} x\left\{\frac { 3 } { 2 } ( 1 - q ) \left[L_{I}(-x)\left(2 A_{0} A(x)+B_{0} B(x)\right)\right.\right. \\
& \left.-L_{Q}(-x) \frac{1}{g}\left(2 A_{0} C(x)-B_{0} D(x)\right)\right] \\
& -\left[L_{I}(-x)(2 A(x)+B(x)-1)\right. \\
& \left.\left.-L_{Q}(-x) \frac{1}{g}(2 C(x)-D(x))\right]\right\}\left(\int_{0}^{1} \mathrm{~d} x L_{I}(-x)\right)^{-1} .
\end{aligned}
$$

\section{The results of calculations}

First of all we consider the dependence of the functions $A(\mu), B(\mu), C(\mu)$, and $D(\mu)$ on the degree of true absorption $q$ and on the parameter $b$ (see Eq. (24)), which characterizes the additional decrease in the Stokes parameters $Q$ and $U$ due to fluctuations of Faraday rotation angles. In Fig. 2 we present the values of these functions at the degree of true absorption $q=0,0.1,0.5$, and $b=0$ and sometimes at $b=100$. Below we give short description of the behavior of these functions, which partly follows from this figure.

The function $A(\mu)$ always grows monotonic with the increase in $\mu$ from the value 0.25 at $\mu=0$. This function acquires its maximal value $A(1)$, depending on the parameters $q$ and $b$. The value $A(1)$ with the increase in parameters $q$ and $b$ diminishes from $A(1)=1.19678$ at $q=0$ and $b=0$ up to 0.5 at $q \rightarrow 1$. The dependence on the parameter $b$ is weak. Thus, for $q=0$ at $b \rightarrow \infty$, the value $A(1) \rightarrow 1.13795$. Function $B(\mu)$ depends on parameter $b$ stronger but mostly near the value $\mu=1$. Thus, for $q=0, B(1)=0.4408$ at $b=0$, and $B(1)=0.5898$ at $b \rightarrow \infty$. At $q \rightarrow 1$ the value $B(1) \rightarrow 0$. For $\mu=0.5$ the corresponding values of $B(0.5)$ are equal to 0.7571 and 0.7142 ; i.e., the deviation is opposite. It is directly seen from system (26) that, in the limit $b \rightarrow \infty$, the functions $A(\mu)$ and $B(\mu)$ obey the separate system of equations, which describes the multiple scattering of radiation with the Rayleigh phase function $(3 / 16 \pi)\left(1+\left(\boldsymbol{n} \boldsymbol{n}^{\prime}\right)^{2}\right)$.

The functions $B(\mu), C(\mu)$, and $D(\mu)$ are non-monotonic - first they slightly increase from $0.5,0.25$, and 0.5 , respectively, at $\mu=0$, and then decrease. The functions $C(\mu)$ and $D(\mu)$ always tend to 0 at $\mu \rightarrow 1$. In the limit $b \rightarrow \infty$, the functions $C(\mu)$ and $D(\mu)$ tend to its free terms $-\left(1-\mu^{2}\right) / 4$ and $\left(1-\mu^{2}\right) / 2$, respectively (see Fig. 2 at $b=100$ ). Because these functions determine the light polarization, it means that at large Faraday's rotation angles the polarization of outgoing radiation is due to the last scattering before the escape from the atmosphere.

The value $2 A(\mu)+B(\mu)-1$ characterizes the integral intensity of radiation from the surface of the whole "spot" (see Eq. (32)). This value hardly depends on the parameter $b \propto \lambda^{4}$, so at $q=0$ we have $2 A(1)+B(1)$ for $b=0$ is equal to 2.8344 , and for $b=50$ is 2.8652 , i.e. the difference is about $1 \%$. At $q=0.5$ these values differ lesser - about $0.1 \%$ (see Fig. 2). This is natural if one remembers that, even in the absence of the magnetic field, the influence of the light polarization on the angular distribution is very weak. In the Milne problem for the scattering according to Rayleigh's phase function one has the value $J(1)=3.02$ at $q=0$ for angular distribution $J(\mu)=I(\mu) / I(0)$. Taking the polarization into account, we obtain $J(1)=3.06$. The high values of $b$ give 
rise to the depolarization of the radiation and, as a result, the radiation intensity is determined by the separate transfer equation with the Rayleigh phase function.

The polarization spectrum is determined by expression (34). Because the value $2 A(\mu)+B(\mu)$ hardly depends on $\lambda$ (see previous paragraph), the spectrum is determined by the value $2 C(\mu)-D(\mu)$. From the second formula (27), one can obtain the asymptotic expression for this value at high values of the parameter $g=1+(1-q) b$. Indeed, at large $g$ the expression $1 /(\mu+g x)$ has a peak-like form with the maximum at $x=0$. At $x \simeq 0$ the functions $A(x)$ and $B(x)$ are described by its free terms (see Eqs. (26)). On the other hand, the functions $C(\mu)$ and $D(\mu)$ for large $b$ also tend to its free terms. As a result, we obtain the following asymptotic formula:

$$
\begin{aligned}
2 C(\mu)-D(\mu) \simeq & \frac{3(1-q) \mu\left(1-\mu^{2}\right)}{16 g} \\
& \times\left[\frac{3}{2}-\frac{3 \mu}{g}-\ln \frac{g+\mu}{\mu}\right] .
\end{aligned}
$$

Here $g=1+\alpha \lambda^{4}$, where the coefficient $\alpha=0.64(1-$ q) $f_{B} \tau_{1}^{(T)}\left\langle B^{\prime 2}\right\rangle / 3$. The logarithmic term in this expression gives large distortion from the simple dependence $p(\lambda) \propto 1 /\left(1+\alpha \lambda^{4}\right)$, especially for relatively low values of $b$, when the polarization degree is rather large and can be registered in the observations. Note that, in the square brackets, we neglect the small term $3 \mu^{2} / g^{2}$. This gives rise to the better approximation of the function $2 C(\mu)-D(\mu)$ for the intermediate values of the parameter $g$. The value (38) is always negative; i.e., the polarization plane coincides with the plane $(\boldsymbol{n N})$.

The comparison of formula (38) with the numerical calculations (at $b>5$ ) shows that the values (38) always are lower than the exact values. The relative difference is practically the same for all $\mu$. At $q=0$ the relative deviations are $\simeq 10 \%, \simeq 20 \%$, and $\simeq 25 \%$ for $b=50,10$, and 5 , respectively. With the increase in $q$ the deviations decrease. At $q=0.5$ they are two times less than at $q=0$. The simple asymptotic formula (38) can be used to approximate the observed polarization spectra and to estimate the parameters $b$ and $\mu$.

The results of calculating of the polarization degree $p(\mu)$ and the polarization spectra $p(\lambda)$ for the case of the point-like source of isotropic non-polarized radiation are presented in Figs. 3-7. Remember that $\mu=\cos \vartheta$, with $\vartheta$ the angle between the normal $\boldsymbol{N}$ and the line of sight $\boldsymbol{n}$ (see Fig. 1).

Figure 3 demonstrates that the polarization $p(\mu)$ is equal to zero at $\mu=1$ and $\mu=0$. In the first case, this is the consequence of the axial symmetry of the problem. In the second case, this is due to the decrease in the observing projection of the "spot" up to zero at $\mu=0$. If one excludes the direct intensity from the source, then at $\mu=0$ the polarization degree will be equal to $p(0)=$ $(3 g)^{-1}$. The case $b=0$ in Fig. 3 corresponds to the absence of magnetic field and coincides with the results of Grinin \& Domke (1971).

The existence of the true absorption $(q \neq 0)$ in an atmosphere decreases the degree of polarization. This occurs due to a relative increase in the part of non-polarized radiation coming directly from the source compared with the partly polarized reflected light that diminishes when $q$ grows (remember that albedo $a \rightarrow 0$ at $q \rightarrow 1$ ).

If one excludes the direct radiation from the source then, in contrast, the polarization degree of outgoing radiation

$p(\mu)=\frac{|2 C(\mu)-D(\mu)|}{2 A(\mu)+B(\mu)-1}$
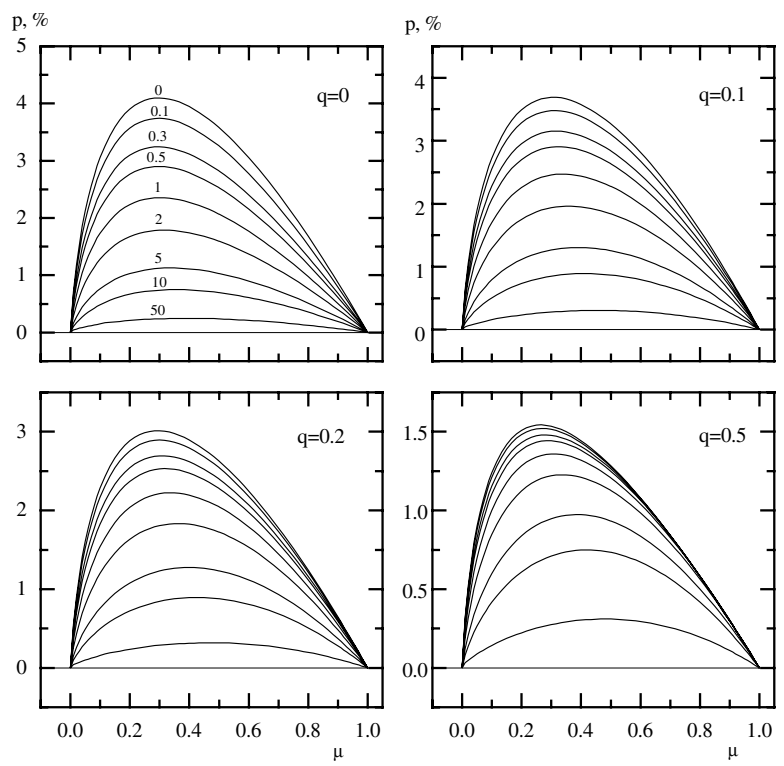

Fig. 3. The angular dependence of the observed polarization degree. The numbers denote the values of the parameter $b=$ $0.64 \tau_{1}^{(\mathrm{T})} f_{B} \lambda^{4}(\mu \mathrm{m})\left\langle B^{\prime 2}\right\rangle / 3$. The curves in other figures correspond to the same values of $b$ and are in the same order.

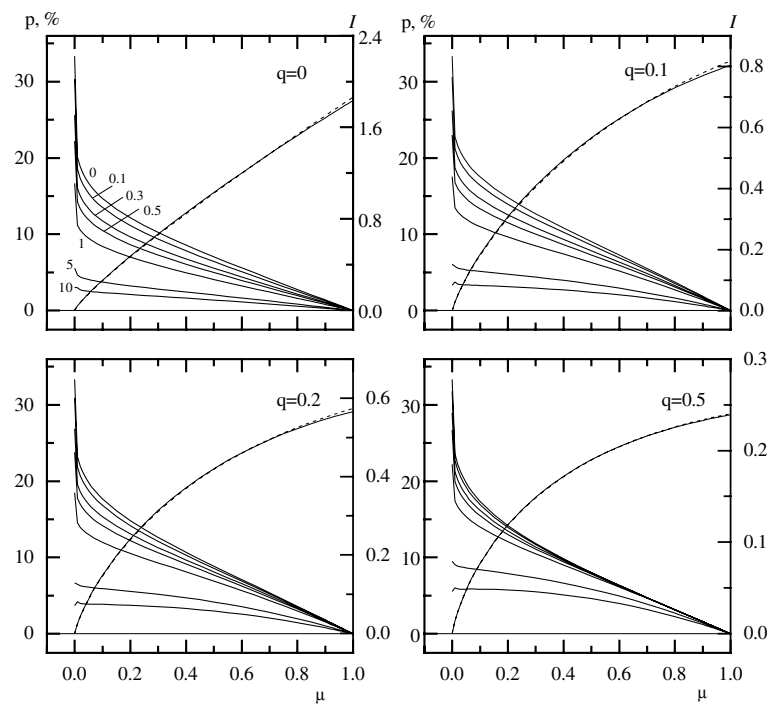

Fig. 4. The intensity $I=2 A(\mu)+B(\mu)-1$ and the polarization degree of scattered radiation without the inclusion of direct non-polarized radiation from the source (see formulae (32) and (39)). The numbers denote the values of parameter $b$ (see Fig. 3).

at $b=0$ first increases with the growth in $q$ and then begins to decrease, staying greater than the value at $q=0$. The deviations from the case $q=0$ are not large, so for $\mu=0.5$ and the values of $q$ equal to $0,0.1,0.2$, and 0.5 the polarization degrees are $10.23 \%, 12.70 \%, 12.62 \%$, and $10.84 \%$, respectively. For this reason, it is difficult to explain this effect qualitatively. It seems that this non-monotonic dependence of the polarization degree is related to the variation in the relative contributions of the parallel and transverse gradients of the radiation density.

The intensity and the polarization degree of radiation without the direct radiation are presented in Fig. 4 . We present there the degree of polarization (39) and the value $I=2 A(\mu)+B(\mu)-1$ for the $b$-parameters equal to $0,0.1,0.3,0.5,1,5$, and 10 . The degree of true absorption, as in Fig. 3, acquires the values 0, 0.1, 

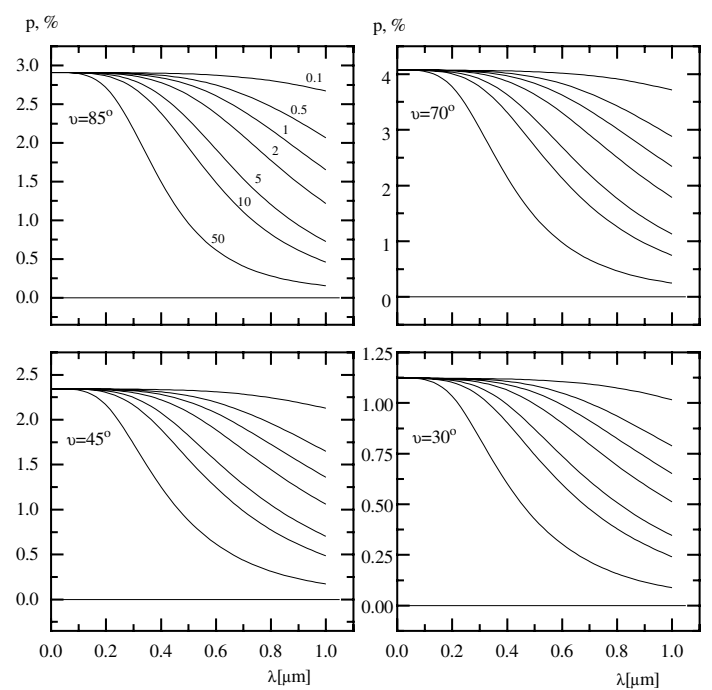

Fig. 5. The spectra of the observed polarization degree $p(\lambda, \vartheta)$ for the angles $\vartheta=85,70,45$, and $30^{\circ}$ for the conservative atmosphere $(q=0)$. The parameter $b \equiv \alpha \lambda^{4}(\mu \mathrm{m})$ where $\alpha=0.64 \tau_{1}^{(T)} f_{B}\left\langle\left(B^{\prime}(G)\right)^{2}\right\rangle / 3$. The numbers denote the value of the parameter $\alpha$. The curves in all figures correspond to this parameter in the same order.
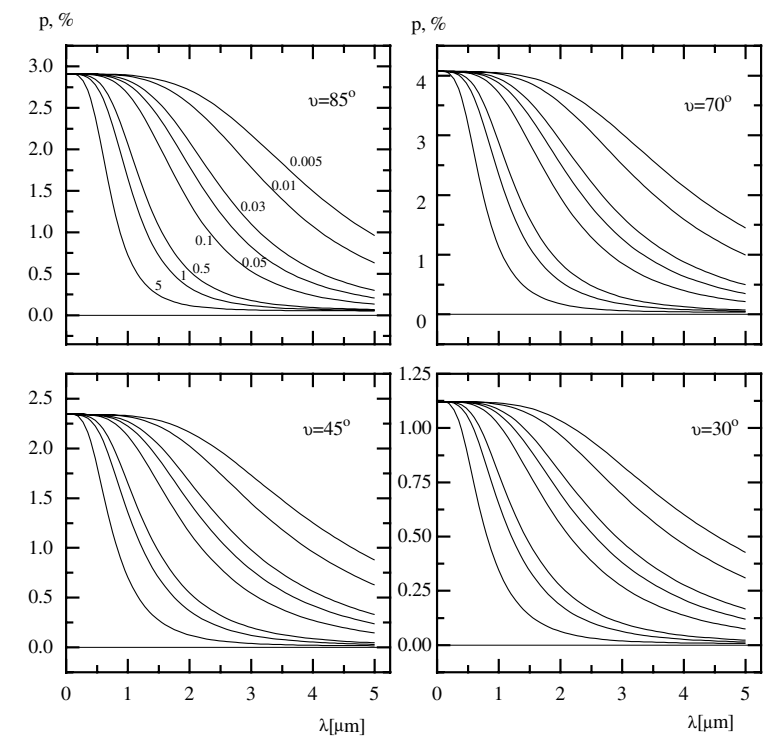

Fig. 6. The same as in Fig. 5 for the wavelength interval $0-5[\mu \mathrm{m}]$.

0.2 , and 0.5 . The dashed curves corresponds to value $b=10$, and solid curves - to $b=0$. It is seen that the value $2 A(\mu)+B(\mu)-1$ is practically independent of $b$. The reason for this effect was discussed earlier.

In Figs. 5-7, the spectra of observed polarization $p(\lambda)$ are presented. The angle $\vartheta$ between the local normal $N$ of the "spot" and the line of sight $\boldsymbol{n}$ takes the values $30^{\circ}, 45^{\circ}, 70^{\circ}$, and $85^{\circ}$. The atmosphere is assumed to be conservative $(q=0)$. In this case the observed polarization is maximal. These spectra can be used to estimate the parameters $b$ and $\mu$.

Our consideration is restricted by the Thomson scattering. The comparison with the known Klein-Nishina formula (see, for example, Haitler 1954) shows that the Thomson approximation is good enough up to the radiation energy $E \leq 10 \mathrm{keV}$ (soft $\mathrm{X}$-rays), when the relative error is $\leq 4 \%$. The results concerning higher energies have the greater errors, so for $E \simeq 50 \mathrm{keV}$ the error is about $20 \%$. For these energies the results presented
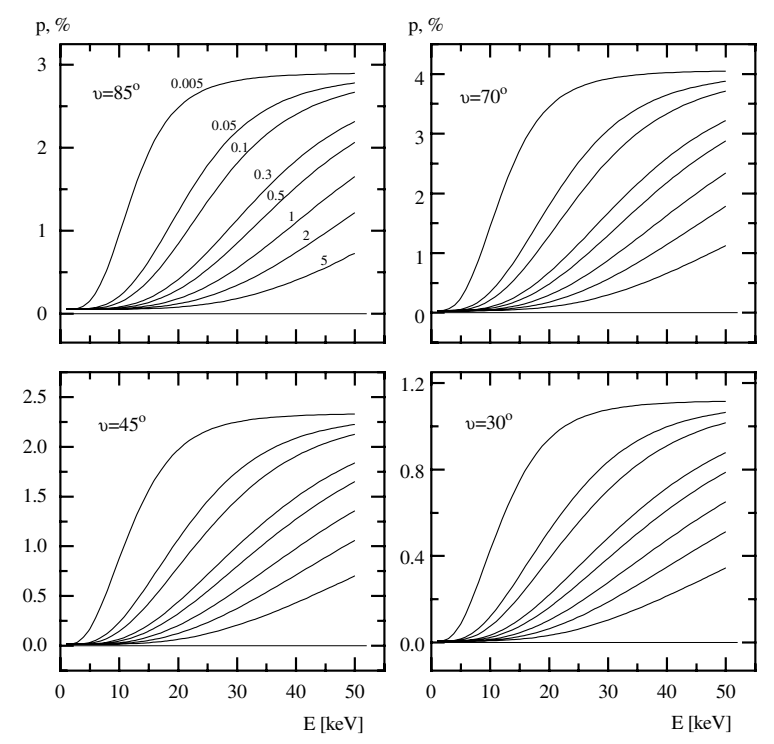

Fig. 7. The same as in Fig. 5 for the photon energy interval $E=1-50 \mathrm{keV}$. The parameter $b \equiv \alpha / E^{4}(\mathrm{keV})$ where $\alpha=$ $0.504 \tau_{1}^{(T)} f_{B}\left\langle\left(B^{\prime} / 10^{6} G\right)^{2}\right\rangle \equiv 50^{4} \beta$. The numbers denote the values of parameter $\beta$, which is numerically equal to $b$ at $E=50 \mathrm{keV}$. The curves in all figures correspond to parameter $\beta$ in the same order.

in Fig. 7 can be used for the preliminary estimates. In the next chapters we discuss mainly the polarization of soft X-rays and the optical radiation where our calculations are fully applicable.

It should be noted that Sobolev's method for considerating the radiation scattered in "spot" can also be used for the Compton scattering because the integration over the surface of a "spot" gives rise to the usual radiative transfer equation for the plane-parallel, semi-infinite atmosphere. The main difficulty in this case is how to solve this equation taking the change of the wavelengths taking into account.

\section{X-ray reflection in accreting black holes}

Studying of the spectra of accreting black holes is a very important task of modern astrophysics. It is especially important for understanding accretion flow and geometry, hence the effects of strong gravity and black hole spin (Ross \& Fabian 2007). Reflection and scattering of X-ray photons on optically thick media are believed to produce features such as the Fe fluorescence emission lines and the broad Compton hump in the spectra of active galactic nuclei (AGN) (e.g. Pounds et al. 1990; George $\&$ Fabian 1991). For example, the detection of a broadened and skewed Fe K-alpha $(E=6.4 \mathrm{keV})$ line in AGN spectra is interpreted as the result of the reflection.

A significant fraction of type I AGN show X-ray excess emission above a power-law continuum identified as the steepening of the X-ray continuum below $\sim 2 \mathrm{keV}$ (Dewangan et al. 2007). Nevertheless, the origin of the soft X-ray emission has remained unclear over the past two decades. One of the popular models, which can explain the observed X-ray excess emission, is the model of ionized reflection from the material surrounding a supermassive black hole.

Unfortunately, the origin of the obscuring material in AGN remains uncertain. Besides accretion flows surrounding the supermassive black hole as the central energetic engine, the photometric and spectroscopical observations show the existence of a dusty geometrically thick obscuring region (the torus), in order to account for many observed differences among various 
classes of AGNs (Antonucci 1993; Chang et al. 2007). This torus may be clumpy, and this situation means that obscuring material presents a turbulent medium.

The polarization is the effective probe to solve the problem of the origin of obscuring material in AGNs. The results of our calculations, presented at Figs. 3, 4, and 7, allow us to estimate the net polarization of reflected X-ray radiation. The degree of polarization is dependent on the angle $\vartheta$ between the local normal $N$ of obscuring material and the line of sight. Figure 7 shows that reflected $\mathrm{X}$-ray radiation is polarized and can reach its maximal value $\sim 4 \%$ for hard ( $E \geq 20 \mathrm{keV}$; if the direct radiation from the source is taken into account) X-ray photons at the angle $\vartheta \sim 70^{\circ}$, and it decreases with the decrease in X-ray energy due to the Faraday depolarization effect. If we consider that the direct radiation from the source is invisible, then the polarization degree is much higher (see Fig. 4) and can acquire the value $\sim 33 \%$. Remember that the turbulent Faraday depolarization effect decreases the polarization degree (with increase of the parameter $b$ ).

Figure 7 also allows us to estimate the polarization of Fe K-alpha line. At the angle $\vartheta \sim 70^{\circ}$ and $b=0.005$, the polarization magnitude is $\sim 1 \%$. For the soft X-ray excess emission, the net polarization is considerably lower: $p<1 \%$. Though the reflection polarization magnitude is not so high (at the level $\geq(0.1-1) \%)$, especially in the soft $\mathrm{X}$-ray region, it will be perfectly measured by the future X-ray Evolving Universe Spectroscopy Mission (XEUS), of course, on condition that the polarization mode in XEUS mission is operated.

Accretion disks in central regions of AGN are subject to strong external illumination originating from some coronal sources. As a result, the specific spectral features appear in the $\mathrm{X}$-ray band. For example, the K-shell lines of iron are found to be prominent around 6-7 keV. It is very important that the shape of the intrinsic spectrum be modified by the strong gravitational field of the central engine (Fabian et al. 2000).

Polarimetric studies could provide additional information about the inner region of an accreting disk that is especially subject to strong gravity regime (Karas et al. 2004; Dovciak et al. 2004; Horak \& Karas 2006). The gravitational field of a black hole influences the Stokes parameters of reflected radiation propagating to a distant observer. The detailed calculations of this effect have been made by Connors et al. (1980), Karas et al. (2004), and Dovciak et al. (2004).

According to these papers, the results of calculating the outgoing Stokes parameters $I, Q$, and $U$ in the local reference frame in the accretion disc are to be integrated over the disk's surface and also integrated along the geodesic paths of photons going toward an observer. The second integration gives rise to the deformation of the spectrum of radiation (reddening) and to the change in the Stokes parameters due to rotation of the polarization plane and the change in the polar angle of accepted radiation compared with our local $\vartheta$-dependence. What is important is that the degree of polarization does not change its value. The results of integration depend on the chosen model of the accretion disc.

Considering small radiating "spot" on the disk's surface (see, for example, Czerny et al. 2004), one can separate the integration over surface of the "spot" and the integration over geodesic line of the photons. In this case we assume that the "spot" is small enough to neglect the gravitational differences inside the spot.

The question arises as to how it is possible to distinguish between two effects of strong gravity and stochastic magnetic field near a black hole surrounding an accretion disk. The reflection component has been computed by Karas et al. (2004), with the help of the Monte-Carlo code. Their results have shown that the polarization of reflected radiation can be as high as thirty percent for small inclinations and small heights of the primary source.

In this case, polarization of the reflected radiation only appears to depend weakly on energy, except in the region close to the iron edge at $\sim 7 \mathrm{keV}$. Another conclusion made by Karas et al. (2004) is that overall polarization increases little with energy, which is due to diminishing radiation from the primary source and to the increasing intensity of reflected radiation with energy, but the intrinsic polarization of the reflected light stays roughly constant.

And in contrast, in our case of turbulent magnetized atmosphere of an accretion disk, the polarization of reflected light itself does not stay constant and essentially decreases with energy decrease via the Faraday depolarization effect. Another essential feature of our effect is the abrupt decrease in the polarization degree at small inclinations from the value of $30 \%$ up to the level $\leq 3 \%$.

The strong gravity effect is especially important for obtaining the polarization images and also for investigating polarization variability, because time-lags and polarization images depend on the focusing effects and the light travel time in a strong gravity field (Horak \& Karas 2006).

There is strong evidence that the origin of soft excess below $2 \mathrm{keV}$ is either reflection or absorption from partially ionized material close to the super massive black hole (Grummy et al. 2006; Done et al. 2007; Fabian 2006). There are two potential geometries for this material: one, seen via reflection, is the accretion disk; the other, seen in absorption, is a wind above the disk (Done \& Nayakshin 2007). Both models produce the $7 \mathrm{keV}$ feature, but produce different polarization states (Gnedin \& Silant'ev 1997; Gnedin et al. 2006).

Polarization is the only test that can really distinguish between these two models. For example, in the case where spherically symmetric wind polarization is zero, unlike for the accretion disk for which the reflected radiation is strongly polarized. The existence of a magnetic field directed perpendicular to the disk surface changes the situation even in the case of a spherically symmetric wind (Gnedin \& Silant'ev 1997). The radiation scattered in the wind also becomes polarized due to the absence of any compensation of polarization directions along and across the magnetic field via the Faraday effect. But wavelength dependence and the orientation of position angles are essentially different (see details in the book by Dolginov et al. 1995; and review by Gnedin \& Silant'ev 1997). Thus the polarization states in the two different models of soft X-ray excess (reflection from the disk or the absorption in the wind from the accretion disk) are very different.

\section{The turbulent accretion disk in NGC 4258: evidence from polarimetric observatons}

NGC 4258 is a very good laboratory to attempt to measure the magnetic field directly in an accretion disk around a supermassive black hole. The magnetic field strength could be directly measured very close to the central black hole (Modjaz et al. 2005). NGC 4258 (M106) is a low-luminosity, weakly active Seyfert II galaxy (Seyfert 1.9). Nuclear continuum and narrow emission lines have been detected in a polarized optical light (Barth et al. 1999). The discovery of a rotating molecular disk emitting $\mathrm{H}_{2} \mathrm{O}$ maser lines provides the unique possibility of tracing the nuclear rotation curve in the inner parsec. NGC is also a natural target for spectropolarimetric investigations, because the accretion disk is nearly edge-on (the inclination angle $i=82^{\circ}-84.5^{\circ}$ ). 
Modjaz et al. (2005) present the results of polarimetric observations at $22 \mathrm{GHz}$ of the water vapor masers in NGC 4258 obtained with the VLA and the GBT. They did not detect any circular polarization in the spectrum indicative of Zeeman-induced splitting of maser $\mathrm{H}_{2} \mathrm{O}$ lines and obtained only upper limits for the toroidal component of the magnetic field in the accretion disk of NGC 4258 at a radius of $0.2 \mathrm{pc}$ at the level $90 \mathrm{mG}$. They also obtained a 1-sigma upper limit of $30 \mathrm{mG}$ on the radial component of the magnetic field at the radius of $0.14 \mathrm{pc}$.

The detection of polarized continuum and line emission from the nucleus of NGC 4258 by Wilkes et al. (1995) and Barth et al. (1999) provides new important information on accretion flows around a supermassive black hole. Unlike most Seyfert nuclei, NGC 4258 has strongly polarized narrow emission lines. The narrow-line polarization ranges from $1.0 \%$ for $[\mathrm{SiII}](\lambda=$ $6716 \AA)$ to $13.9 \%$ for the [OII] $(\lambda=7319 \AA, 7331 \AA$ blend $)$, and the position angle of polarization is oriented nearly parallel to the projected plane of the accretion disk. It is an interesting fact that the observed polarization of continuum emission ( $\lambda=4000-4800 \AA, 5100-6100 \AA$ and 7500-8500 $\AA$ ) is too low, at $p_{l}=0.3 \%$, for the edge-on accretion disk (Barth et al. 1999). The expected degree of polarization for inclination angle $i \sim 84^{\circ}$ is equal to $7.5 \%$. Such low polarization of the continuum can be explained in the framework of the model of the turbulent magnetized accretion disk that produces the depolarization via the Faraday effect (Silant'ev 2007). Namely, calculations made by Silant'ev (2007) show that continuum polarization at the level $\sim 0.3 \%$ can be obtained for the turbulent disk with isotropic magnetic fluctuations for turbulent parameter $b>10$. In this case, the stochastic Faraday rotation with isotropic fluctuations efficiently decreases the amplitude of the polarization of each individual beam as it travels through the turbulent atmosphere.

A higher degree of polarization of line emission can be explained by the effect of the reflection of emission of line sources located above the accretion disk. Figures 3-7 show that it is easy to obtain reflection polarization at the level $\sim(1-3) \%$. The higher polarization, $\sim 10 \%$, can be obtained only in the case where the direct line emission is blocked by a molecular torus surrounding the central energy engine, together with the source of a highly polarized emission line.

We suggest consider the situation in NGC 4258 in the separate paper in more detail.

\section{Conclusion}

We obtain the solution to the problem of the reflection of the radiation from point-like sources of anisotropic and polarized light that is located over the turbulent magnetized plasma atmosphere. The calculation of the integral Stokes parameters of outgoing radiation is presented for the case when the regular part of magnetic field is negligible compared with the action of magnetic fluctuations (see the inequality (23)). These integral parameters are observed by the telescope from the spot-like part of the atmosphere below the point-like source of the radiation. We studied the dependence of the polarization degree on the values magnetic fluctuations and the true absorption in the atmosphere.

The various spectra of polarization are presented for a number of angles between the line of sight and the normal to the "spot" for a conservative atmosphere when the polarization is maximal. Our general solution is also valid for any types of falling bounded radiation, in particular, for the bounded beam of plane-parallel radiation and for the case of bounded sources inside the atmosphere.
The results of calculations can be used when analyzing of the polarization spectra from the binary systems, flare stars, AGN,etc. We discuss in detail the X-ray observations of some AGN and possible values of the polarization degrees for them. The observed polarization of radiation from the object NGC 4258 is explained using our results.

Acknowledgements. This research was made with financial support by the RFBR (project No. 07-02-00535-a) and Program of Prezidium of the Russian Academy of Sciences "Origin and Evolution of Stars and Galaxies", and the program of the Department of Physical Sciences of RAS "Extended Objects". The authors are very grateful to the anonymous referee for many useful remarks.

\section{References}

Agol, E., \& Blaes, O. 1996, MNRAS, 282, 965

Agol, E., Blaes, O., \& Ionescu-Zanetti, C. 1998, MNRAS, 293, 1

Ambartzumyan, V. A. 1942, Sov. Astron., 19, 1

Antonucci, S. 1993, ARA\&A, 31, 473

Blaes, O. M. 2003, in Les Houches Session LXXVIII, 2002, Accretion discs, jets and high energy phenomena in astrophysics, ed. V. Beskin, et al. (N.Y.: Springer), 147

Chandrasekhar, S. 1950, Radiative transfer (Oxford: Clarendon Press)

Chang, P., Quataert, E., \& Murrey, N. 2007, ApJ, 662, 94

Cherepashchuk, A. M. 2000, Ap\&SS, 274, 159

Connors, P. A., Piran, T., \& Stark, R. F. 1980, ApJ, 235, 224

Czerny, B., Rozanska, A., Dovciak, M., Karas, V., \& Dumont, A.-M. A\&A, 420, 1

Devangan, G., Giffiths, R. E., Dasgupta, S., \& Rao, A. R. 2007, [arXiv: astro-ph/0709.1962]

Dolginov, A. Z., Gnedin, Yu. N., \& Silant'ev, N. A. 1995, Propagation and polarization of radiation in cosmic media (N.Y.: Gordon \& Breach)

Done, C., \& Nayakshin, S. 2007 [arXiv: astro-ph/0701141]

Done, C., Gierlinsky, M., Sobolewska, M., Schruch, N. 2007, The Central Engine of Active Galactic Nuclei, ASP Conf. Ser., 373, 21

Dovciak, M., Karas, V., \& Matt, G. 2004, MNRAS, 355, 1005

Fabian, A. C., Iwasawa, K., Reynolds, C. S., \& Young, A. J. 2000, PASP, 112, 1145

Fabian, A. C. 2006, Astron. Nachr./AN 999, 88, 789

[arXiv: 0711.2976]

George, I. M., \& Fabian, A. C. 1991, MNRAS, 249, 352

Gnedin, Yu. N., \& Silant'ev, N. A. 1997, Basic mechanisms of light polarization in cosmic media (N.Y.: Hartwood Academic Publ.)

Gnedin, Yu. N., Silant'ev, N. A., \& Shternin, P. S. 2006, Astron. Lett., 32, 39

Grinin, V. P., \& Domke, H. 1971, Astrophysics, 7, 2112

Grummy, J., Fabian, A. C., Gallo, L., \& Ross, R. R. 2006, MNRAS, 365, 1067 Gurzadyan, G. A. 1980, Flare stars (Oxford: Pergamon Press)

Heitler, W. 1954, The quantum theory of radiation (Oxford: Clarendon Press)

Karas, V., Dovciak, M., \& Matt, G. 2004, 22nd Texas Symposium on relativistic Astrophysics at Stanford University.

Modjaz, M., Moran, J. M., Kondratko, P. T., \& Greenhill, L. J. 2005, ApJ, 626, 104

Pounds, K. A., Nandra, K., Stewart, G. C., George, I. M., \& Fabian, A. C. 1990, Nature, 344, 132

Ross, R. R., \& Fabian, A. C. 2007 [arXiv: 0709. 0270]

Shternin, P. S., Gnedin, Yu. N., \& Silant'ev, N. A. 2003, Astrophysics, 46, 350

Silant'ev, N. A. 1979, Sov. Astron., 23, 21

Silant'ev, N. A. 1994, JQSRT, 52, 207

Silant'ev, N. A. 2002, A\&A, 383, 326

Silant'ev, N. A. 2005, A\&A, 433, 1117

Silant'ev, N. A. 2007, Astron. Rep., 51, 67

Sobolev, V. V. 1963, A treatise on radiative transfer (N.Y.: Van Nostrand) (the russian edition: 1956, Perenos luchistoi energii v atmosferah zvezd i planet, Gostehizdat, Moskwa)

Sobolev, V. V. 1969, Course in theoretical astrophysics, National Aeronautics and space administration, Washington, D.C., NASA TT F 531

Varshalovich, D. A., Khersonsky, V. K., \& Moskalev, A. N. 1988, Quantum Theory of Angular Momentum (Singapure: World Scientific)

Wilkes, B. J., Schmidt, G. D., Smith, P. S., Mathur, S., \& McLeod, K. K. 1995, ApJ, 455, L13 